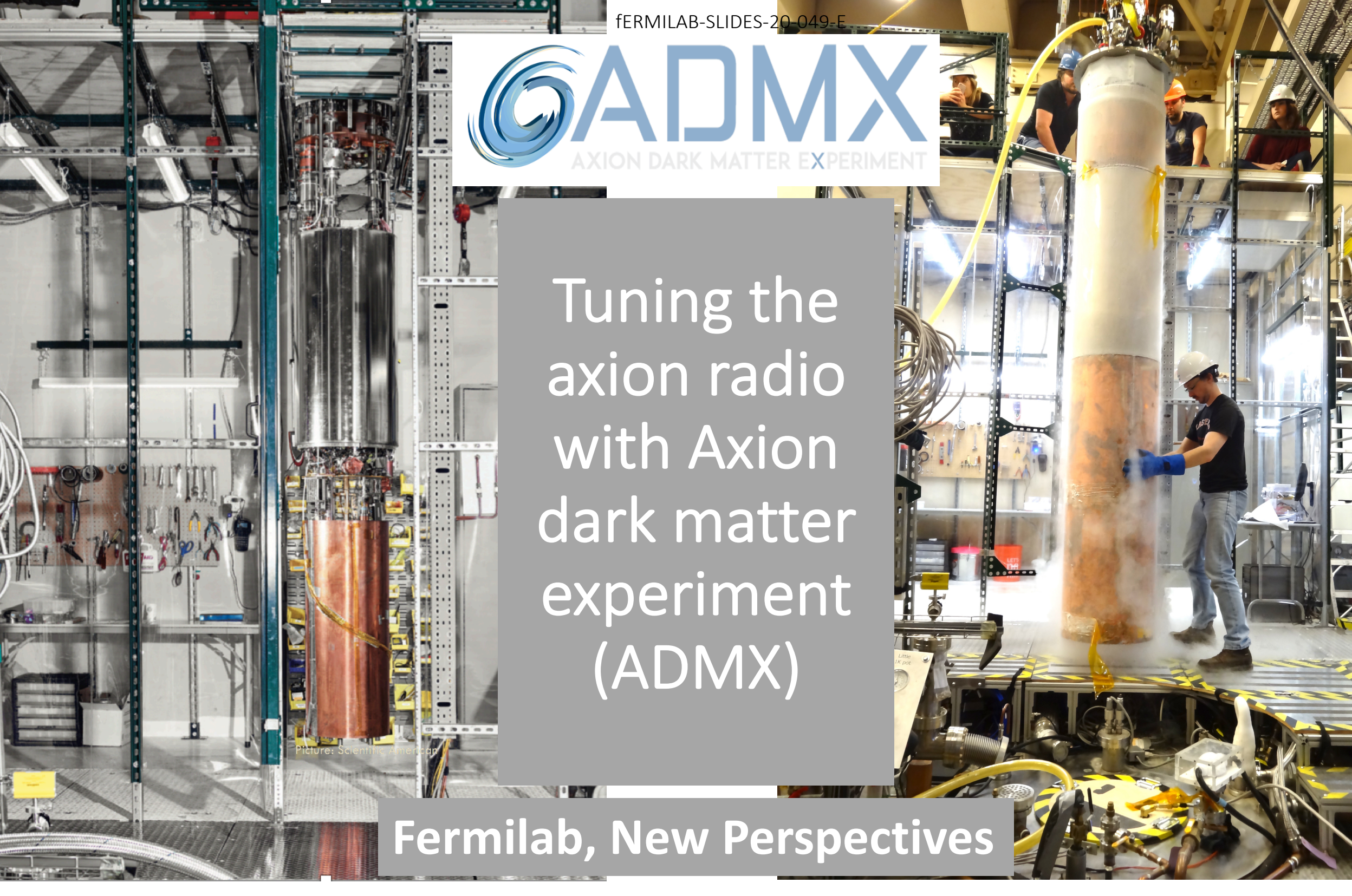




\section{Dark Matter}

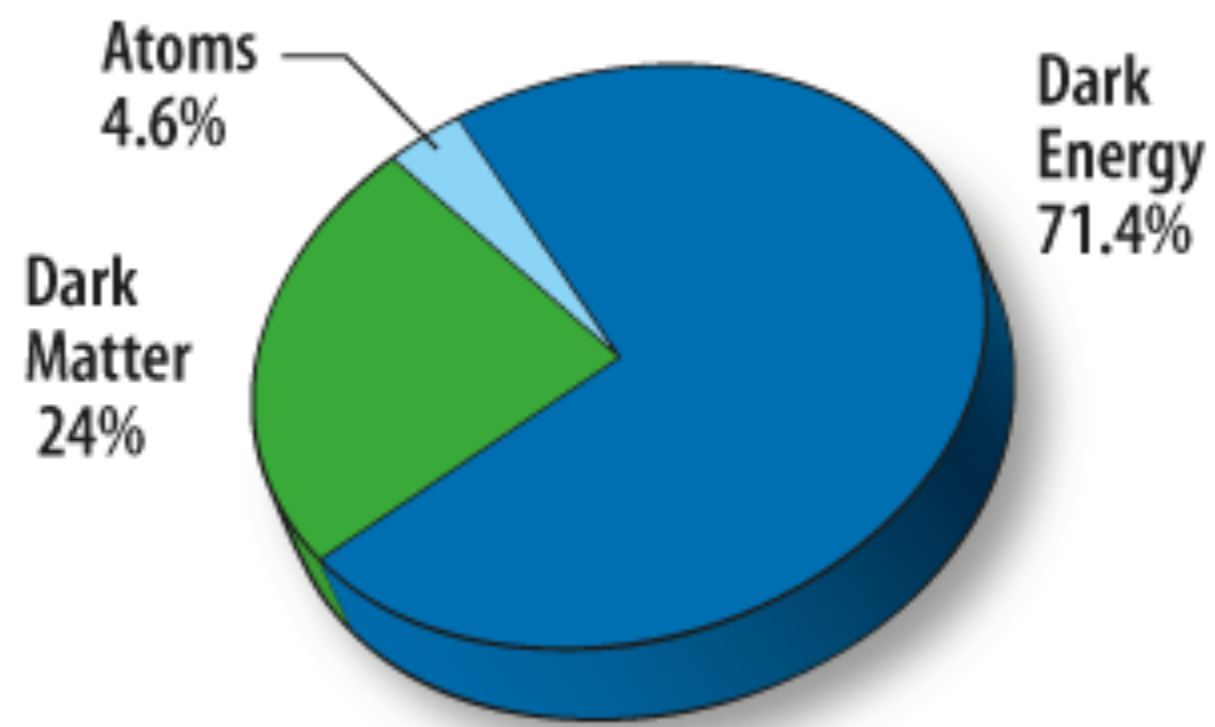

\section{Properties:}

* Non-standard model particle *Weakly interacting - can't be detected with traditional observational astronomy tools doesn't reflect, absorb or emit light

* Makes up large structures of the universe - forms clumps

- cold dark matter

*Axions will be the lightest particle

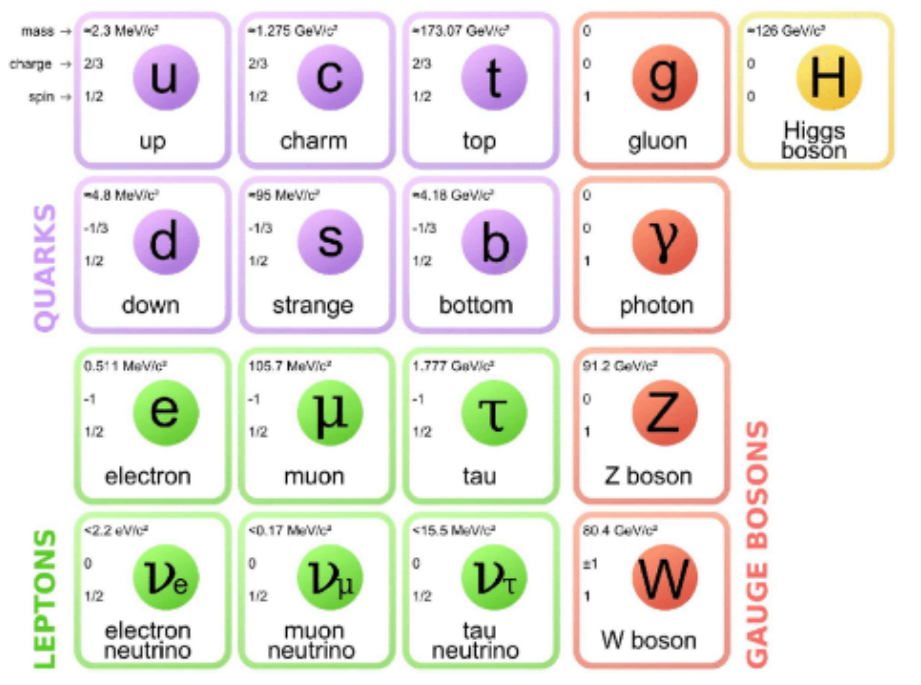




\section{Evidence of dark matter}

*Gravitational lensing: Light bent by galaxies
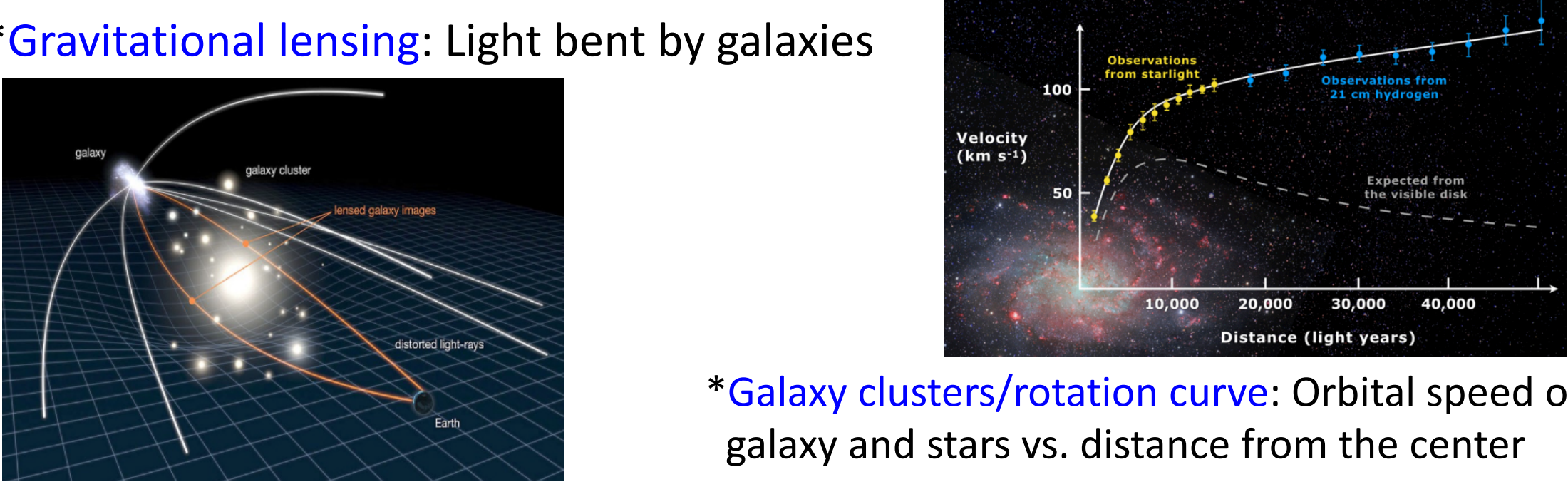

*Galaxy clusters/rotation curve: Orbital speed of galaxy and stars vs. distance from the center

* Shape of the CMB power spectrum:

fluctuation of the $C M B$ temperature vs.

angular scale - indicates existence of dark

matter

*Comparison of model with dark matter and observation matches

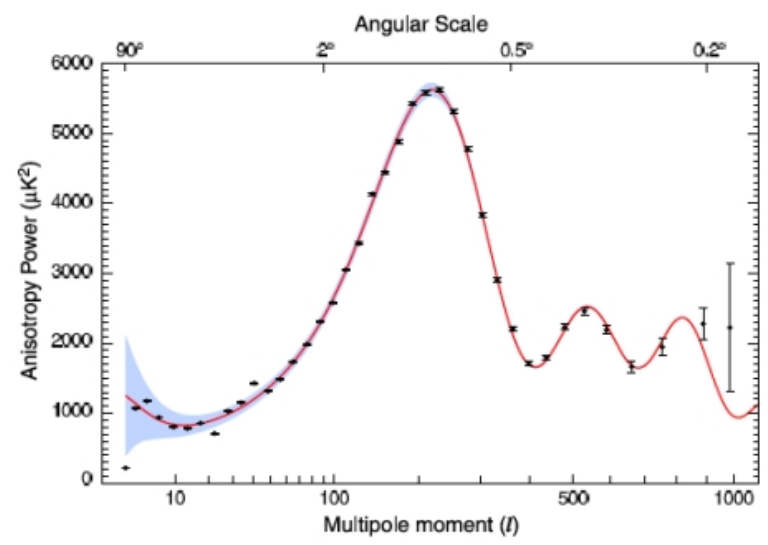




\section{Dark Matter parameter space}
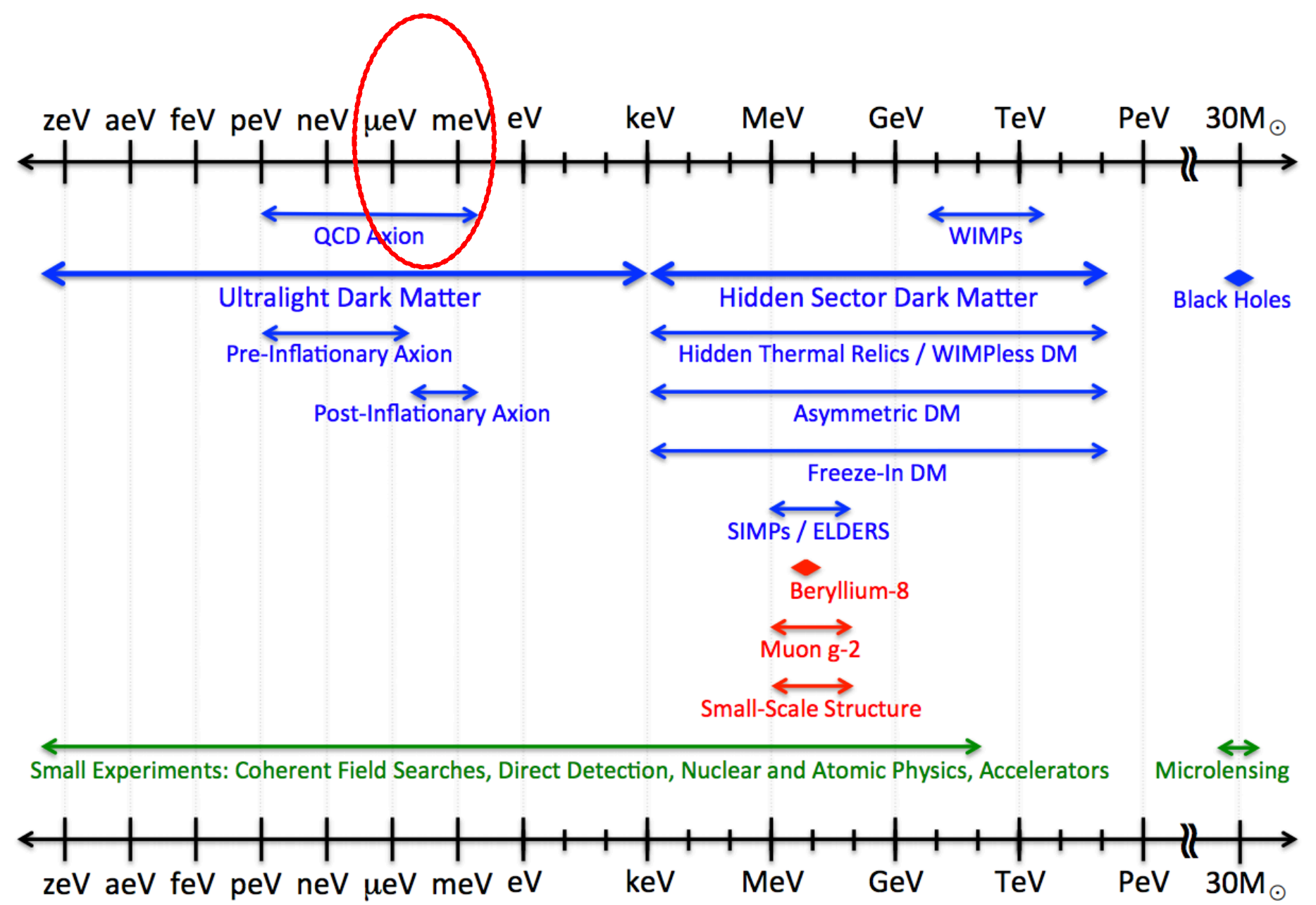

US Cosmic vision 


\section{Axion}

\section{Big Bang}

$\rightarrow$ Axion produced $\sim$ inflation

$\rightarrow$ Theoretically motivated

-- Strong CP problem

$\rightarrow$ Standard Model QCD -- CP violating parameter $\theta(0-2 \pi)$

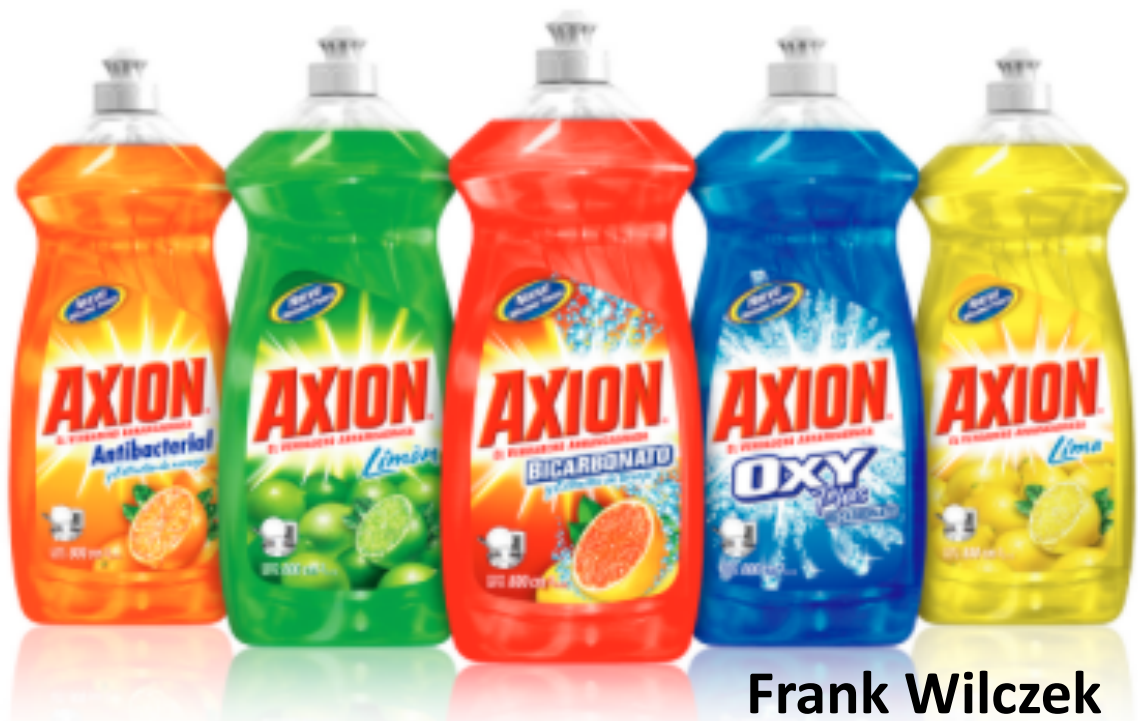

$\rightarrow \theta \neq 0 \Rightarrow C P$ violation in Strong Int. $=>$ neutron's electric dipole moment $d_{n} \neq 0$

$\rightarrow$ Experimental upper limit on $d_{n}$ very small

$=>\theta$ really really small $!=>$ Strong $\mathrm{CP}$ problem

$\Theta$ promoted to a field (Peccei-Quinn theory)

--adding new $\mathrm{U}(1)$ global symmetry to the SM--that gets spontaneously broken

$\rightarrow$ Axion associated particle 


\section{Axion in the galactic halo}
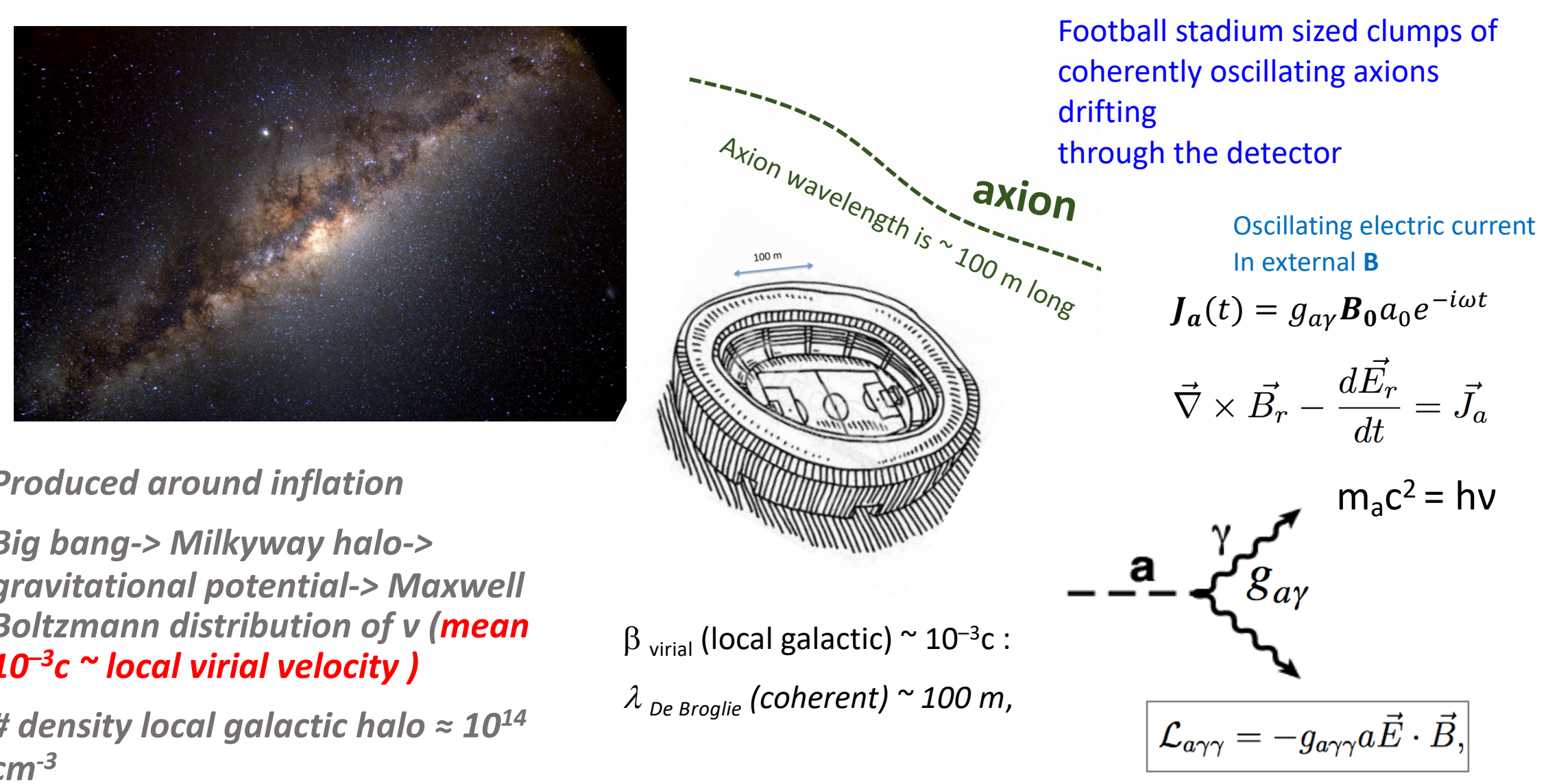

- Produced around inflation

- Big bang-> Milkyway halo-> gravitational potential-> Maxwell Boltzmann distribution of $v$ (mean $10^{-3} \mathrm{c} \sim$ local virial velocity )

- \# density local galactic halo $\approx 10^{14}$ $\mathrm{cm}^{-3}$

Football stadium sized clumps of drifting through the detector

Oscillating electric current In external B

$$
\begin{aligned}
& \boldsymbol{J}_{\boldsymbol{a}}(t)=g_{a \gamma} \boldsymbol{B}_{\mathbf{0}} a_{0} e^{-i \omega t} \\
& \vec{\nabla} \times \overrightarrow{B_{r}}-\frac{d \overrightarrow{E_{r}}}{d t}=\overrightarrow{J_{a}}
\end{aligned}
$$

-- $\left(\rho=450 \mathrm{MeV} / \mathrm{cm}^{3}\right)$

$\square$ Lifetime $10^{42}$ years! 


\section{A good axion detector}

$\square$ Tunable in frequency (compton) mass of axion unknown

$\square$ Low thermal photon background $=>$ very cold

$\square$ Low added electronics noise => quantum technology

ADMX $=>$ World's most sensitive RF receiver

*Sensitivity: $10^{-26}$ Watts *A cellphone with similar capability: 4 bars on Mars!!

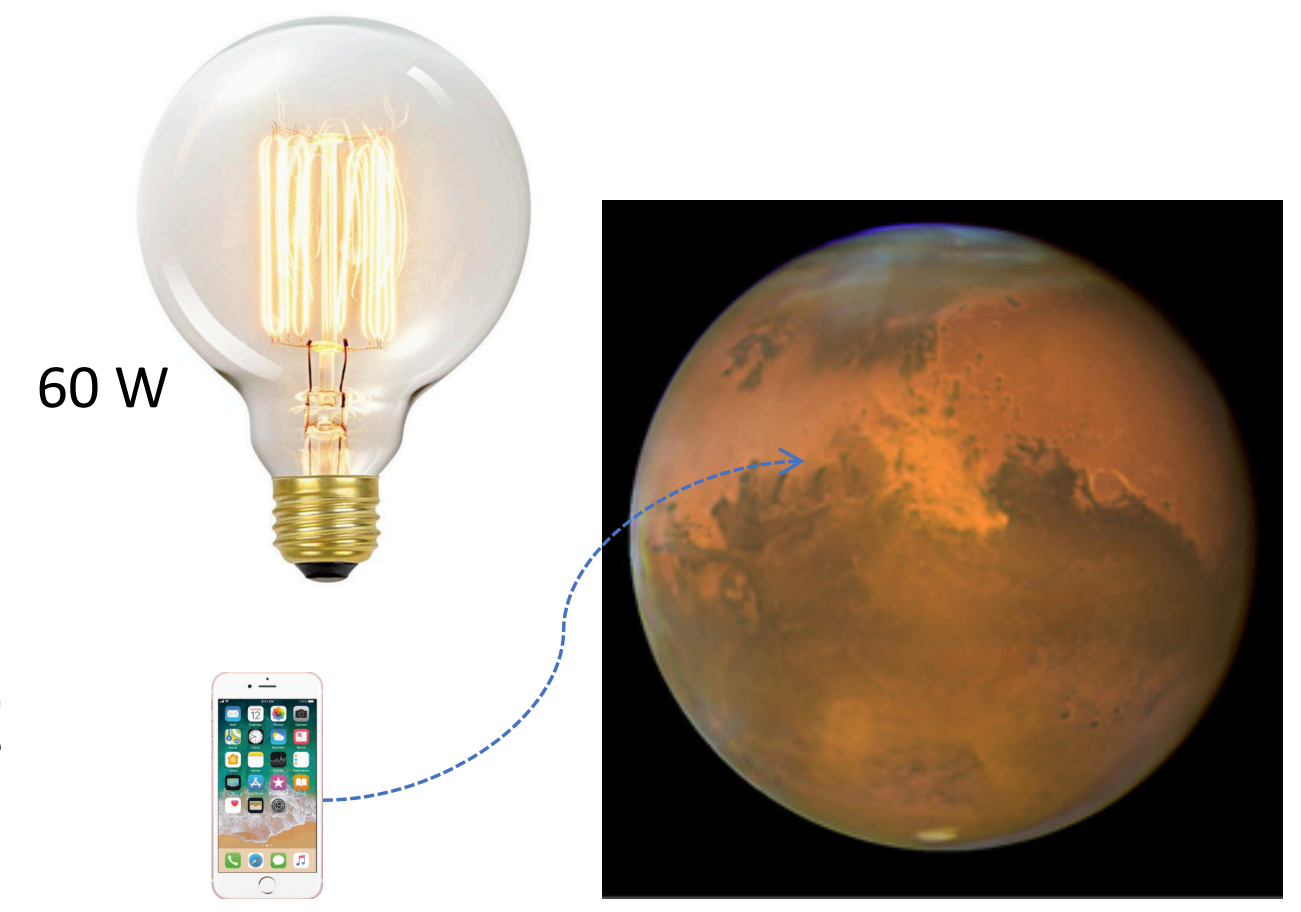




\section{How to detect axion?}

- Analogous to radio tuning.
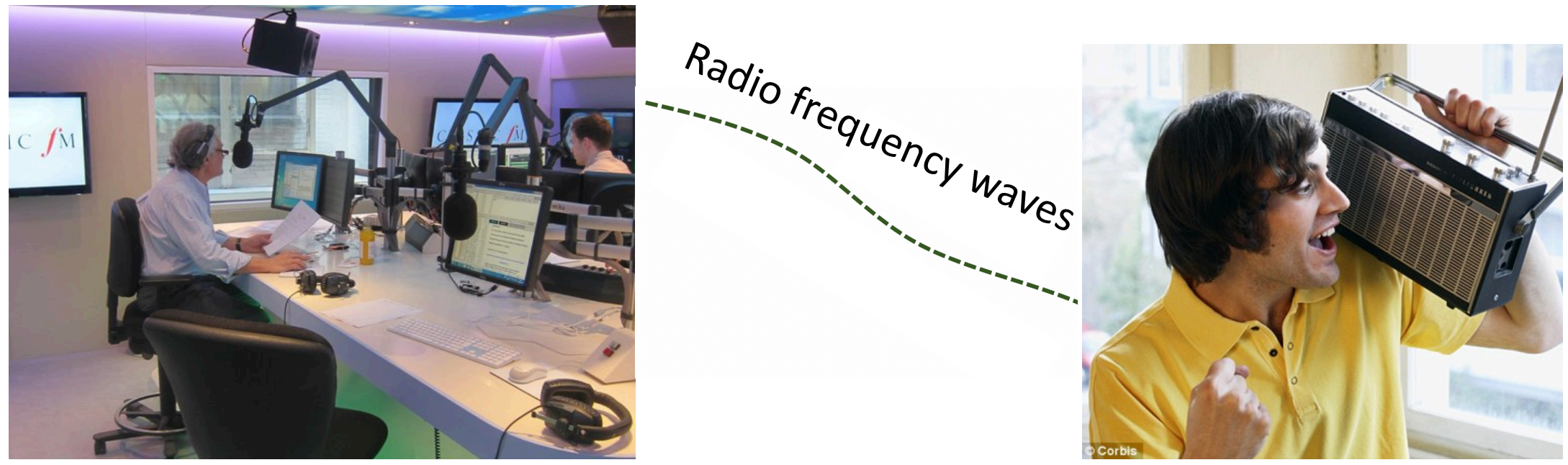

Radio station

When your radio's (electronics) frequency matches to that of the broadcasted FM's frequency, you can hear the music 


\section{How to detect axion?}

- Analogous to radio tuning.

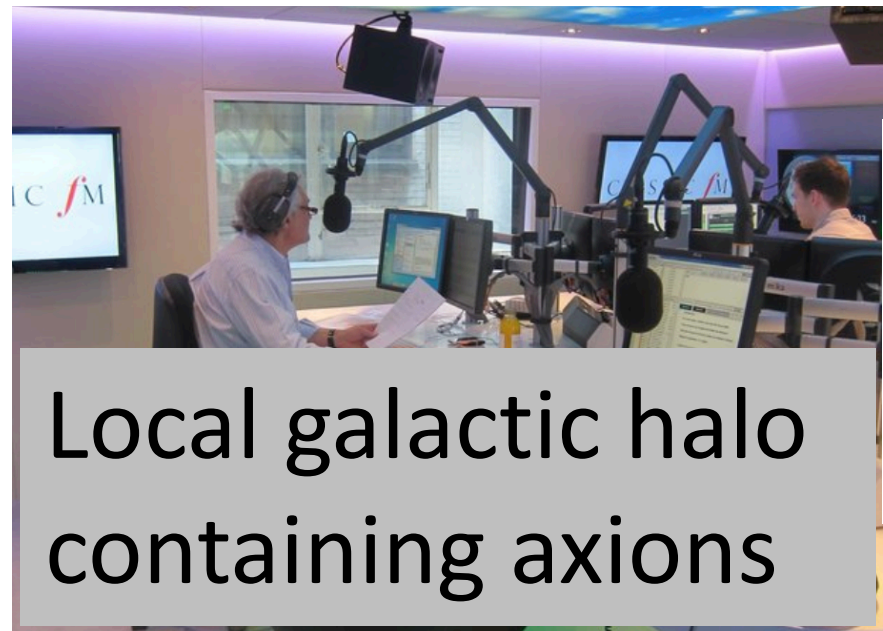

Radio station

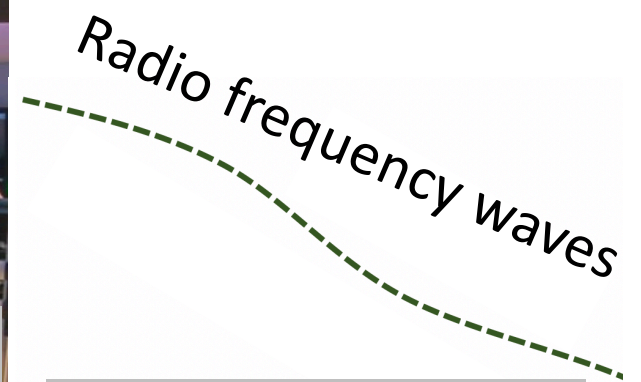

\section{axion wave}

electronics

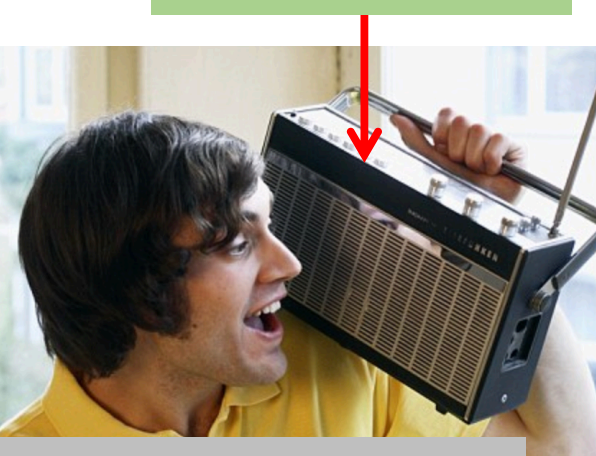

Haloscope experiment

When your haloscope's frequency matches to that of the axion's frequency, you can detect the axion (if it exists) 


\section{Axion dark matter radio}

\section{The Axion Haloscope}

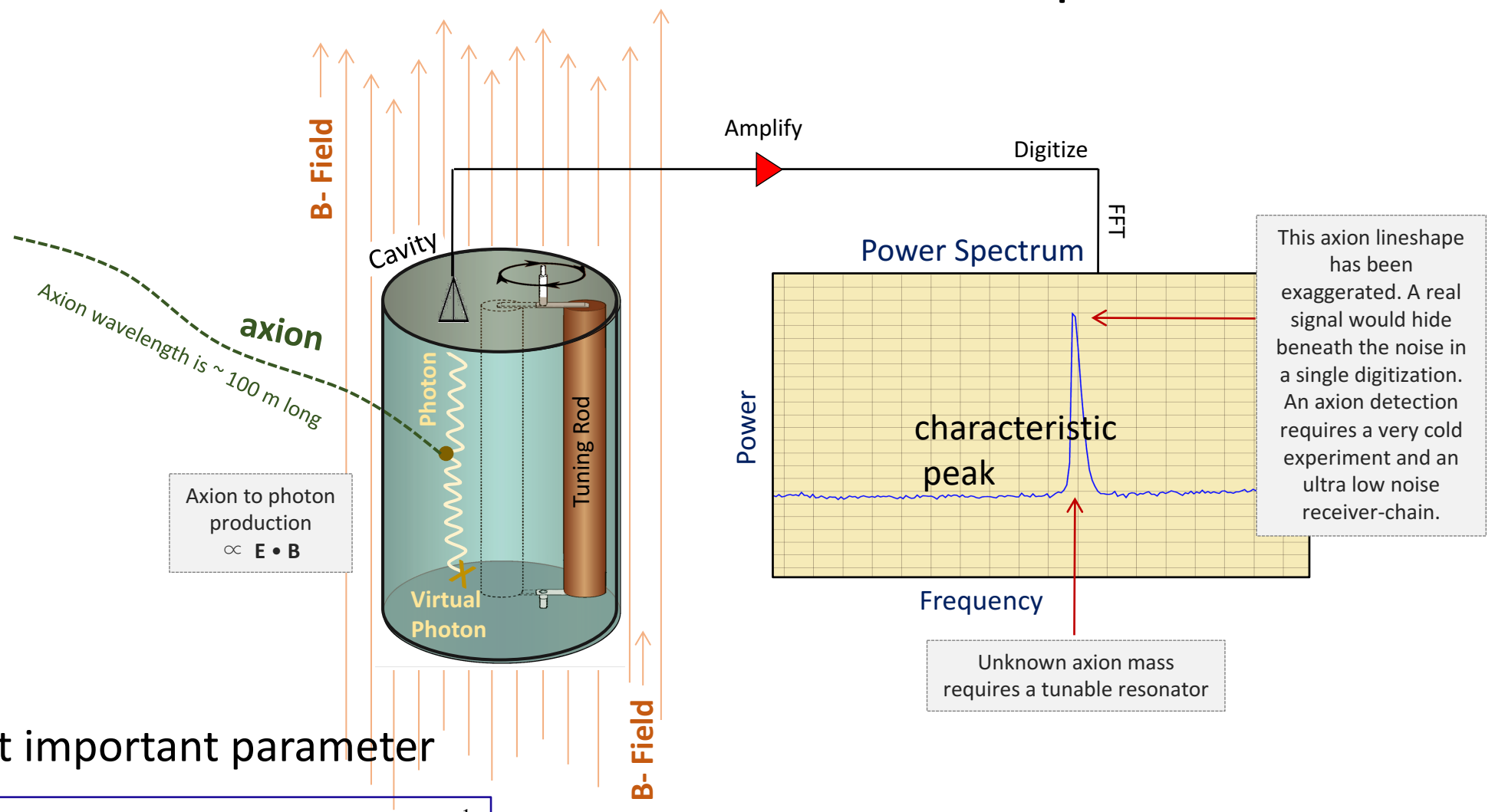

$S N R \propto \frac{P_{\text {out }}}{k_{B} T_{\text {system }}} \sqrt{\frac{t}{b}} \propto \frac{g_{\text {ay }}^{2} \rho_{a} f Q C_{m n p} B^{2} V t^{\frac{1}{2}}}{b^{\frac{1}{2}} T_{\text {system }}}$ 


\section{Searching for a tiny signal}
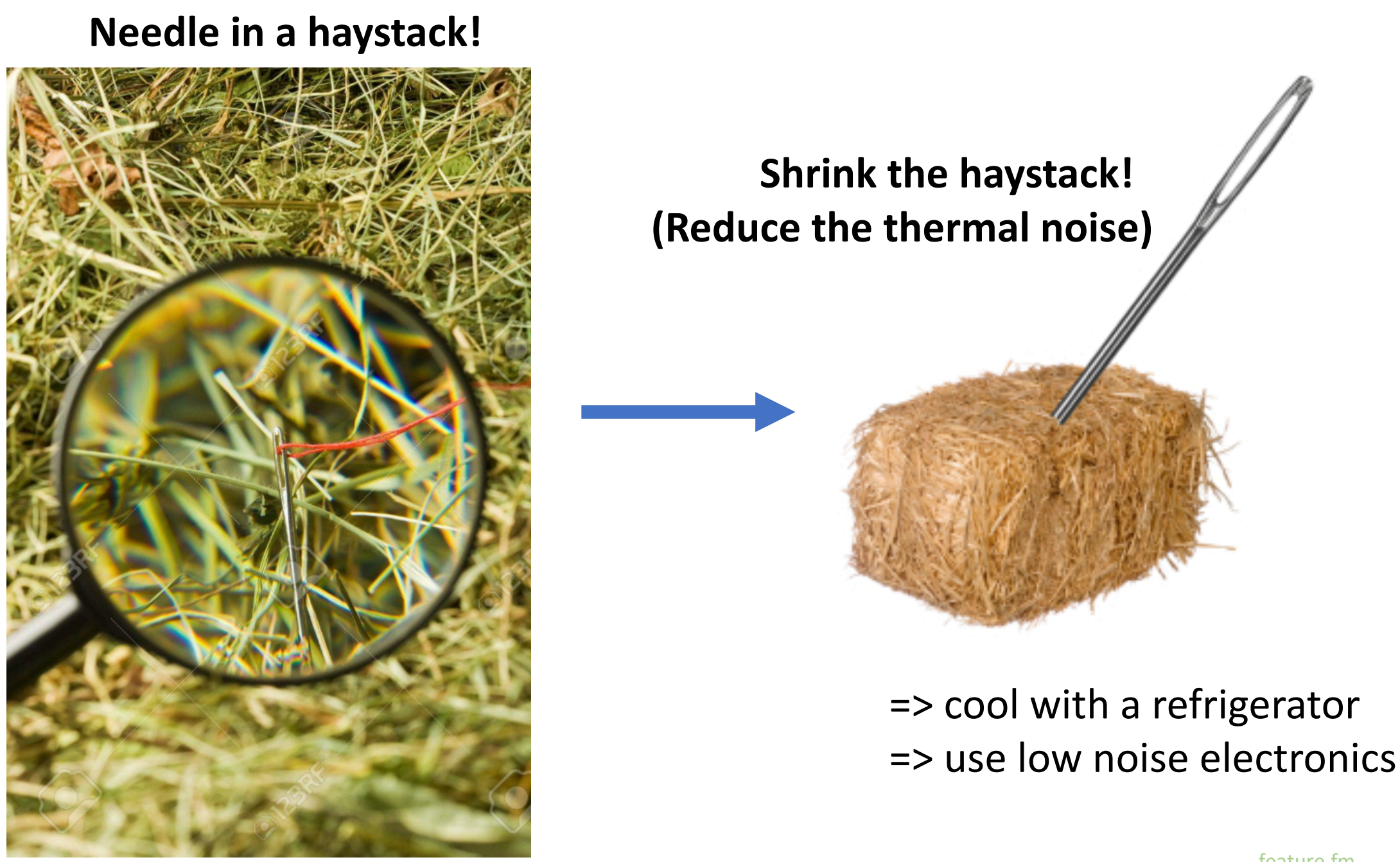


\section{ADMX detector}
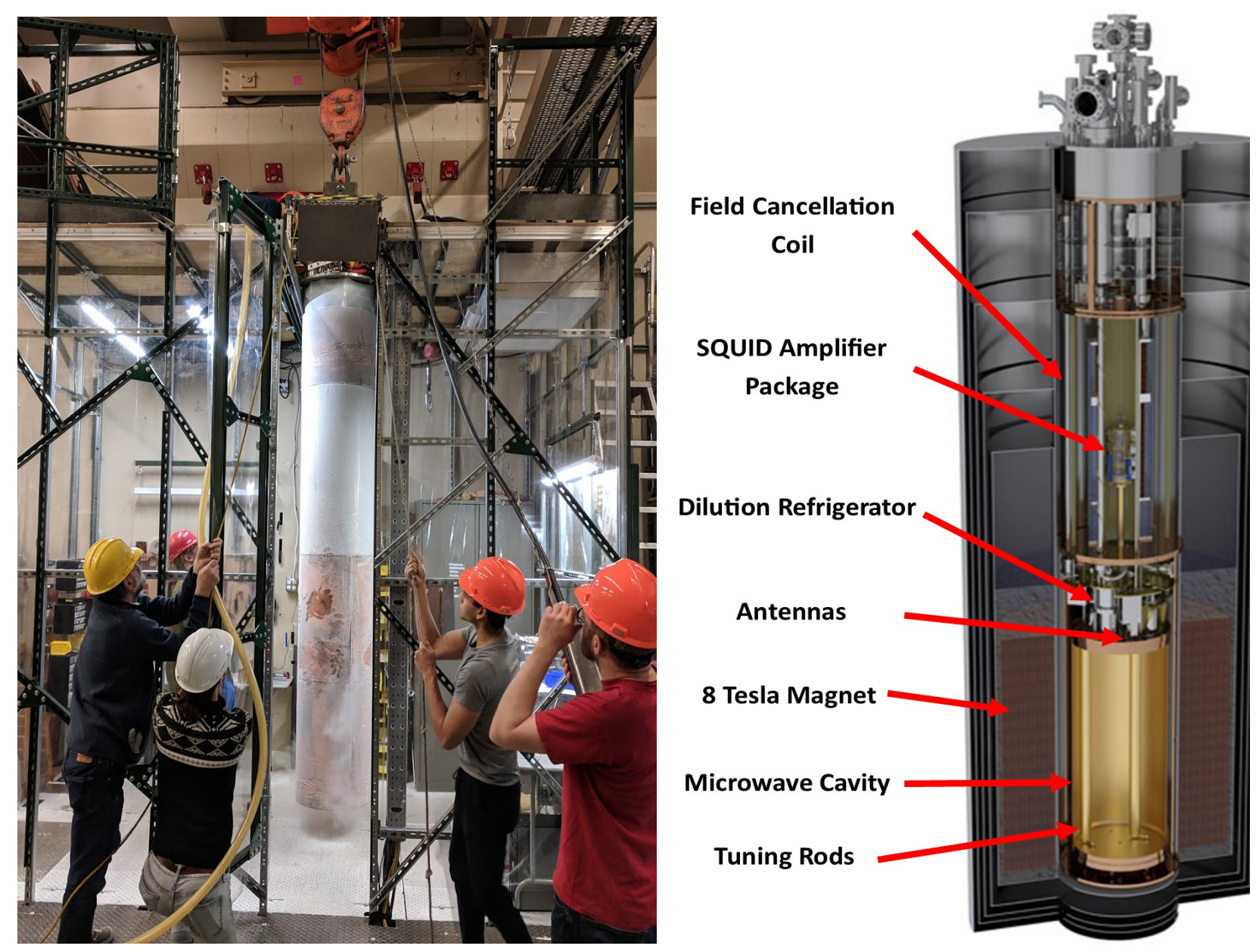

Field cancellation coil: cancels the residual magnetic field around the SQUID electronics

\section{Superconducting} QUantum Interference Device (SQUID) amplifiers: amplifies the signal while being quantum noise limited

Dilution refrigerator: cools the insert to $\sim 90 \mathrm{mK}$

Antennas: pick up signal

Magnet: facilitates the axion conversion to photons, $8 \mathrm{~T}$

Microwave Cavity: converts axions into photons, tunable 


\section{ADMX results 2018-2020}

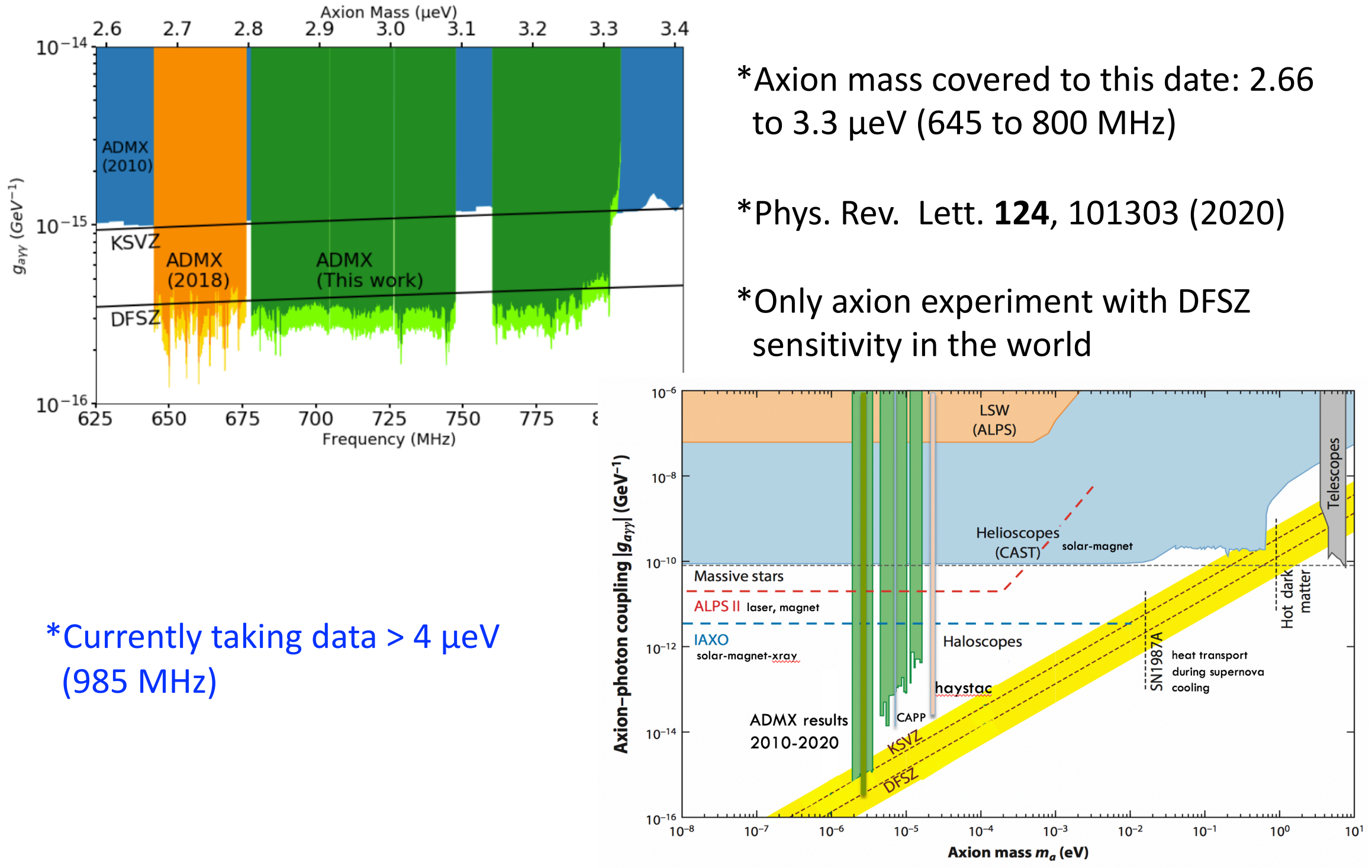




\section{What would an Axion signal look like?}

- Synthetic Axion Generator (SAG)--software simulated axion signal added to real data

- -weighted signal by Lorentzian line shape

- Combined added spectra

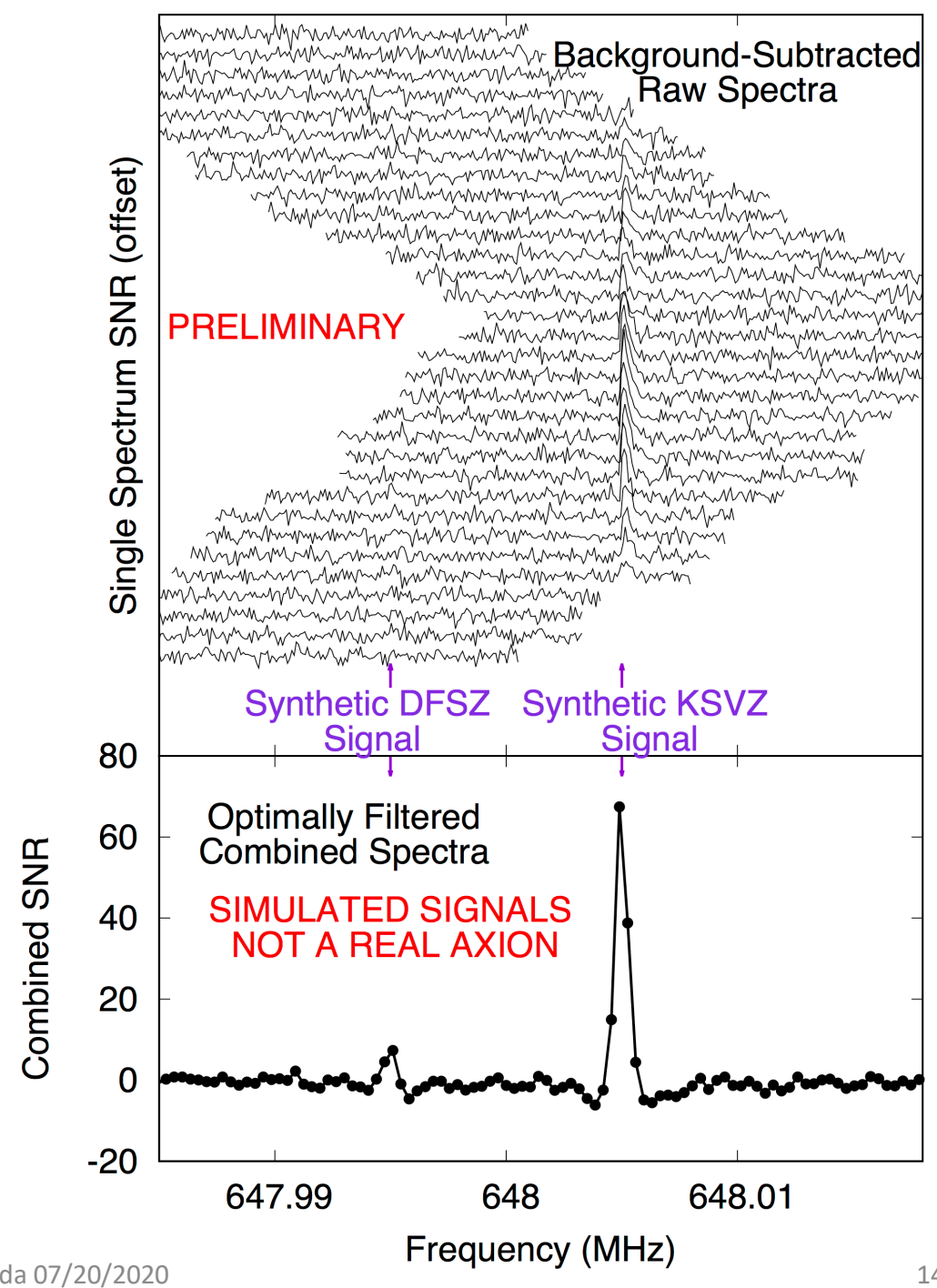




\section{Future direction: key parameters}

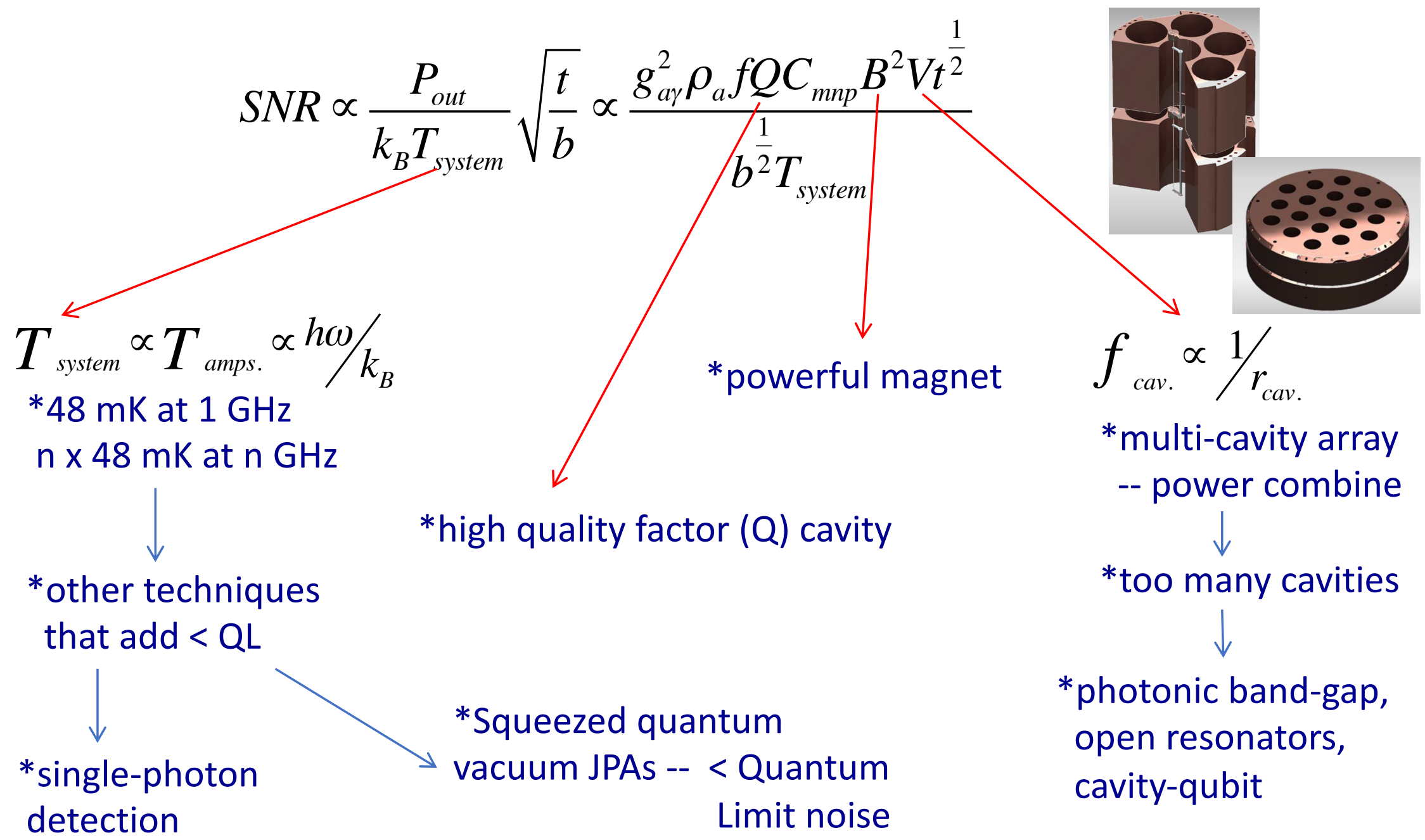




\section{Axion search summary}

- ADMX DFSZ sensitivity -- forefront of Axion Dark Matter search

- If discovered, axions will:

-- tell us about early universe

-- solve the strong CP problem

-- solve the Dark Matter puzzle

- Future direction:

-- quantum science based novel methods and technology

-- without these, axion search impossible in reasonable amount of time

- DISCOVERY CAN HAPPEN ANYTIME DURING DATA TAKING! - 2020 run ongoing! (>4 $\mu \mathrm{eV}$ axion) - Stay tuned!! 
GADMX

\section{Collaboration}

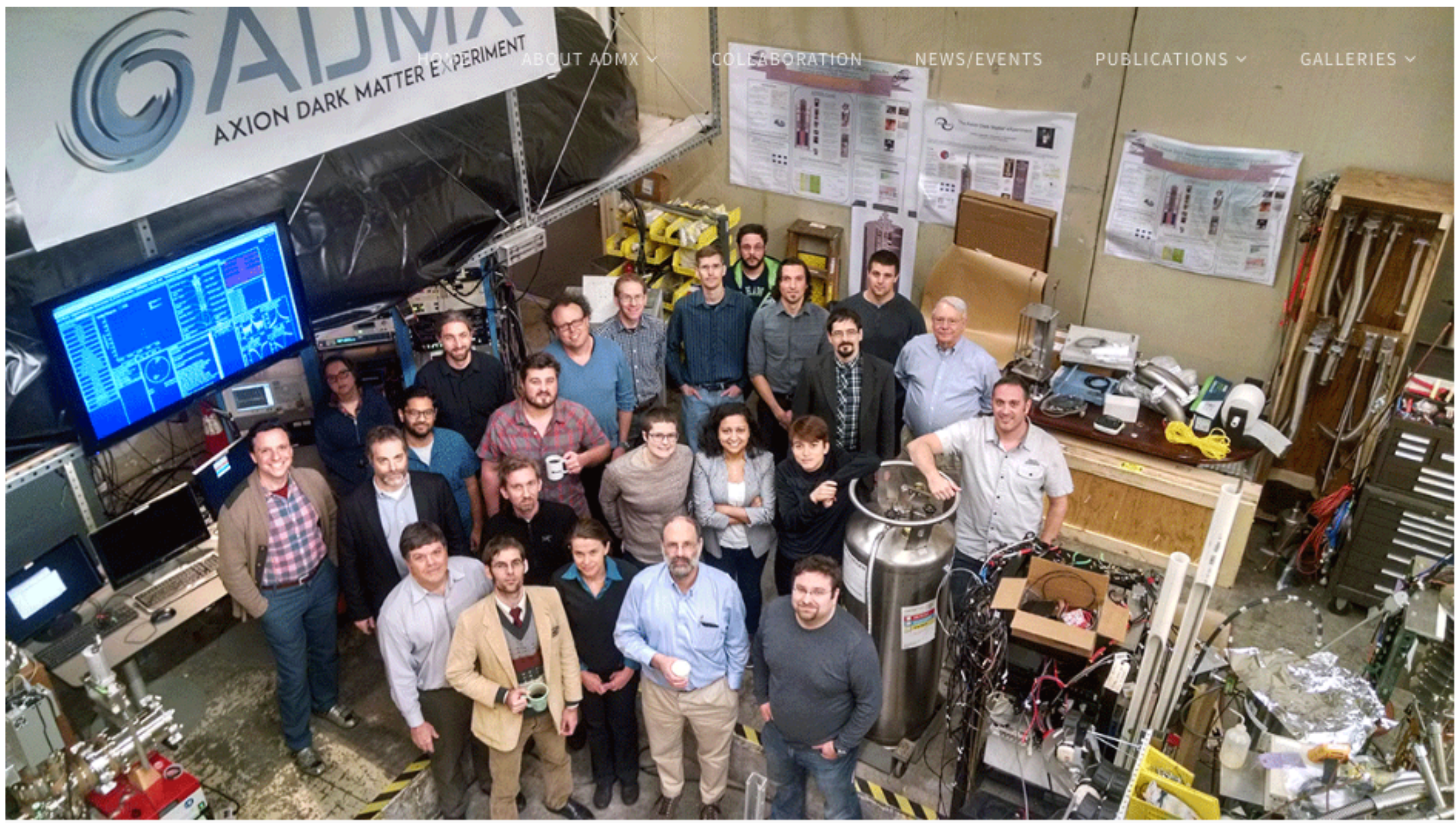




\section{Acknowledgement}

\section{U.S. Department of Energy, Office of High-Energy \\ Physics contract DE SCO011665 \&}

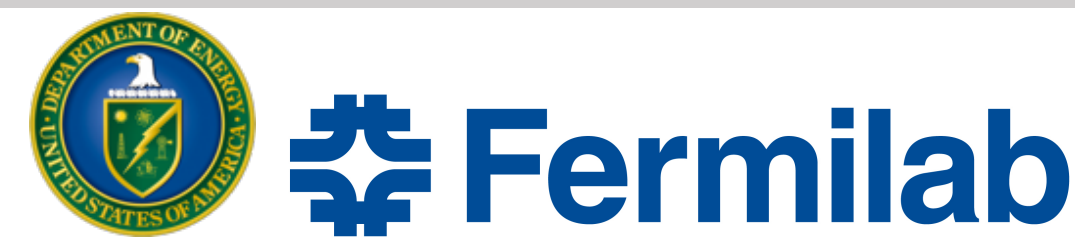

This work was supported by the U.S. Department of Energy through Grants No. DE-SC0009723, No No. DE-SC0010280, No. DE-SC0010280, No. DEFG02-97ER41029, No. DE-FG02-96ER40956, No. DE/ 07NA27344, and No. DE-C03-76SF00098. Fermilab is a U.S. Department of Energy, Office of Scienc Facility. Fermilab is managed by Fermi Research Alliance, LLC (FRA), acting under Contract No. DE-A 07CH11359. Additional support was provided by the Heising-Simons Foundation and by the Lawrer National Laboratory and Pacific Northwest National Laboratory LDRD offices.

\section{Heising-Simons Foundation}

University of Washington, Dept. of Physics

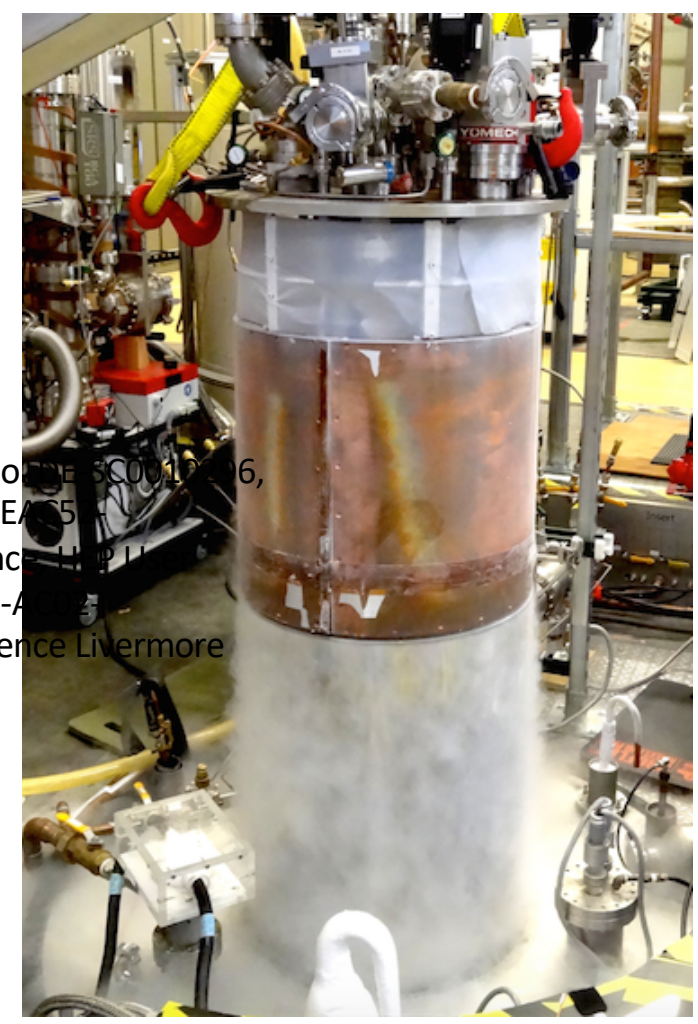




\section{Additional slides}




\section{Axion}

where the amplitude of the axion wave

$$
\theta_{0}=\sqrt{\frac{2 \rho_{a}}{\Lambda_{\mathrm{QCD}}^{4}}} \approx 3.7 \times 10^{-19} \text { radians }
$$

$P_{\text {axion }}=1.9 \times 10^{-22} \mathrm{~W}\left(\frac{V}{136 l}\right)\left(\frac{B}{6.8 \mathrm{~T}}\right)^{2}\left(\frac{C}{0.4}\right)\left(\frac{g_{\gamma}}{0.97}\right)^{2}\left(\frac{\rho_{\mathrm{a}}}{0.45 \mathrm{GeV} \mathrm{cm}^{-3}}\right)\left(\frac{f}{650 \mathrm{MHz}}\right)\left(\frac{Q}{50,000}\right)$. 


\section{Axion production}

- Global symmetry broken at scale $f_{a}$

-- axion produced through misalignment mechanism

-- during QCD phase transition, trough tilted by $\Lambda_{\mathrm{OCD}}{ }^{4}$

- $\mathrm{PE} \sim \Lambda_{\mathrm{QCD}}{ }^{4}$ released, makes up dark matter

-- oscillation of the QCD $\theta$ angle about its minimum--vacuum energy to axions

- QCD axion mass $m_{a} \sim \Lambda_{Q C D}{ }^{2} / f_{a}$

--- $f_{a}$ unknown

$\sim(200 \mathrm{MeV})^{2} / \mathrm{f}_{\mathrm{a}}$

$\Rightarrow \mathrm{GHz}$ frequencies at $\mathrm{f}_{\mathrm{a}} \sim 10^{13} \mathrm{GeV}$ scale
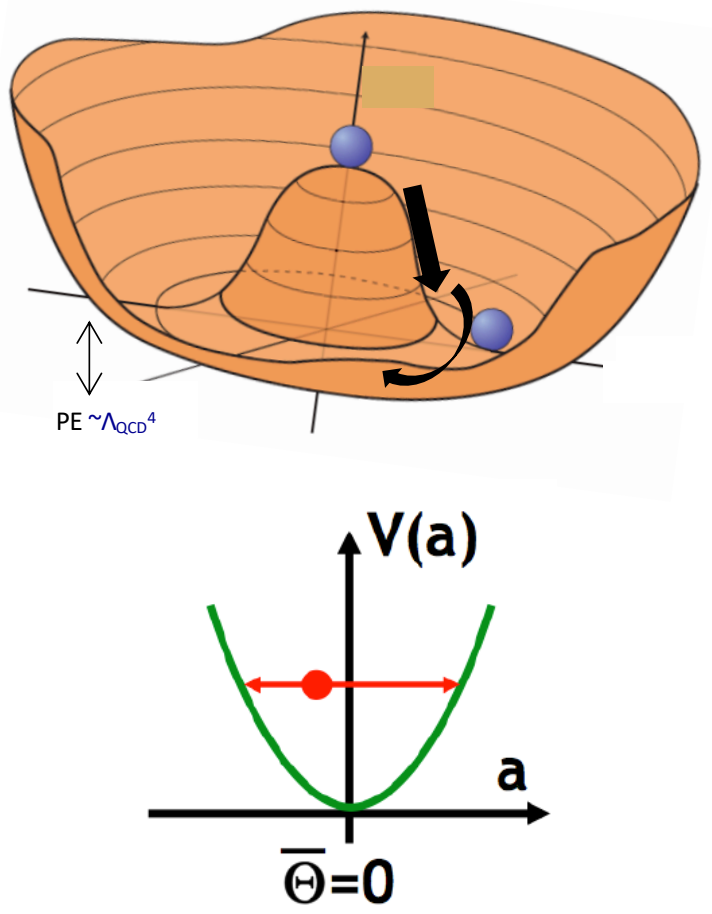

Fig 1:J. Ellis et al; arxiv:1201.6045v1 


\section{SAG}

- Blind injection - input fake axion signal (python script) to arbitrary function generator mixed with local oscillator to axion like frequencies 


\section{Noise temp.}

$$
\begin{aligned}
& T_{N, M S A}=T_{\text {sys. }}-T_{H F E T} \\
& T_{H F E T}=T_{N, H F E T} / G_{M S A} \\
& T_{\text {syst. }}=T_{N, H F E T} / S N R
\end{aligned}
$$




\section{Data Taking/Analysis steps}

- Tune the cavity resonance $\mathrm{TM}_{010}$ to the desired mass of Axion (photon frequency), tune the SQUID amps. to match this.

- NA checks at this frequency: antenna coupling, $Q_{\text {cav }}$

- SA (Digitize): Record noise power spectra data for $100 \mathrm{~s}$ in a BW of $25 \mathrm{kHz}$ centered at $\mathrm{TM}_{010}$

- For one bin with this BW $(25 \mathrm{kHz})$, use at least 20 overlapping noise power spectra

- Background receiver transfer function shapes were removed to $95 \%$ of least-deviant power bins using Savitsky Golay filter shapes (length 121, polynomial order 4) removes signal much broader than axions.

- Power scaled to known $T_{\text {sys }}$ and weighted by $Q_{L}$ to produce excess power in each bin for Axion signal

- This excess power is then convolved using two astrophysical signal shapesMaxwellean predicted by Standard Halo Model and N-body shape.

- When the data were statistically consistent with no Axion signal, the Power equation is used to put the limits on the coupling.

- Frequencies with $>3 \sigma$ above the mean power were flagged candidates for rescan/analysis

- If persists, individually checked for RF interference 


\section{$\mathrm{N}$-body line-shape}

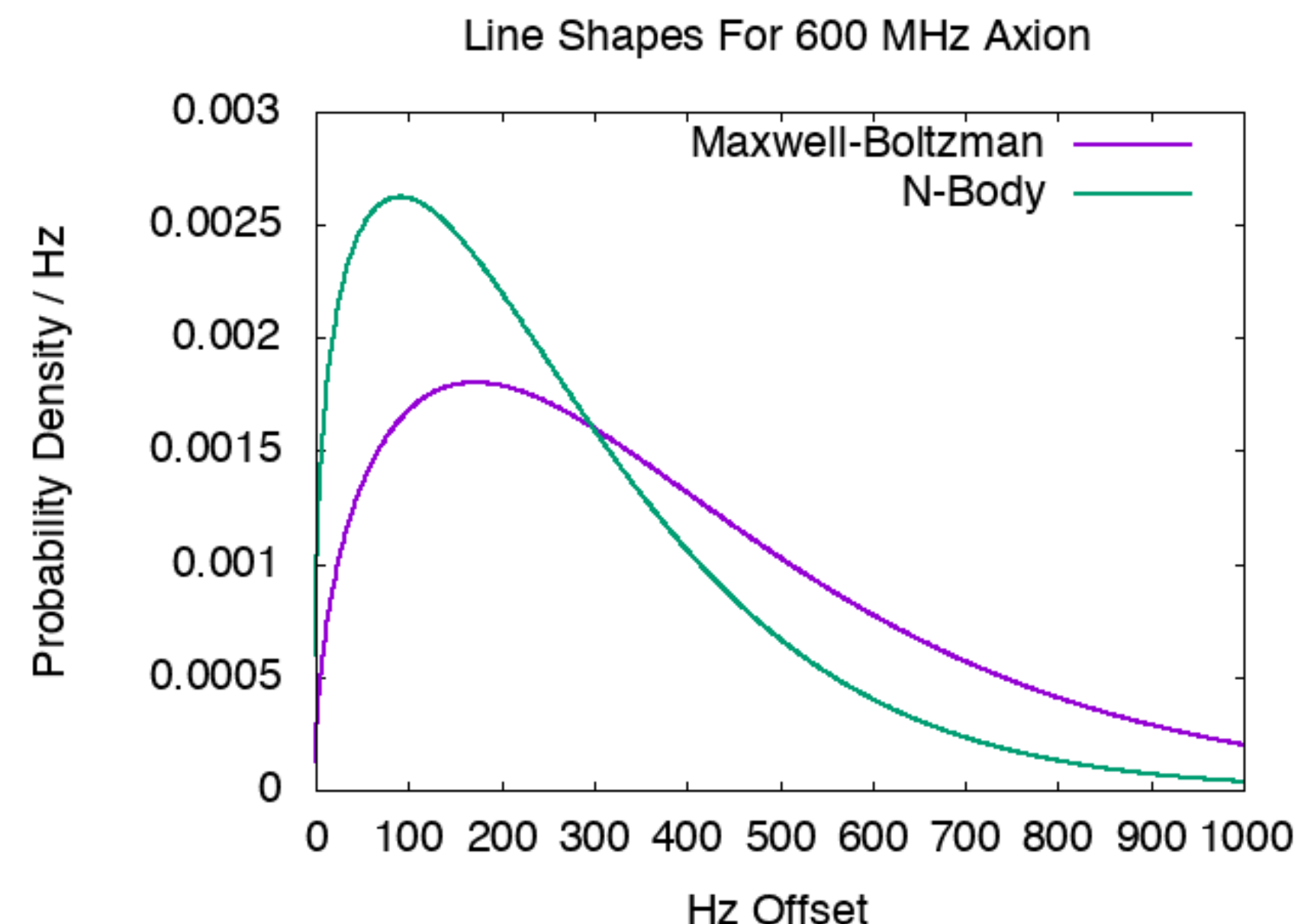




\section{Power transfer increased by coherence between cavity E-field and axion field}

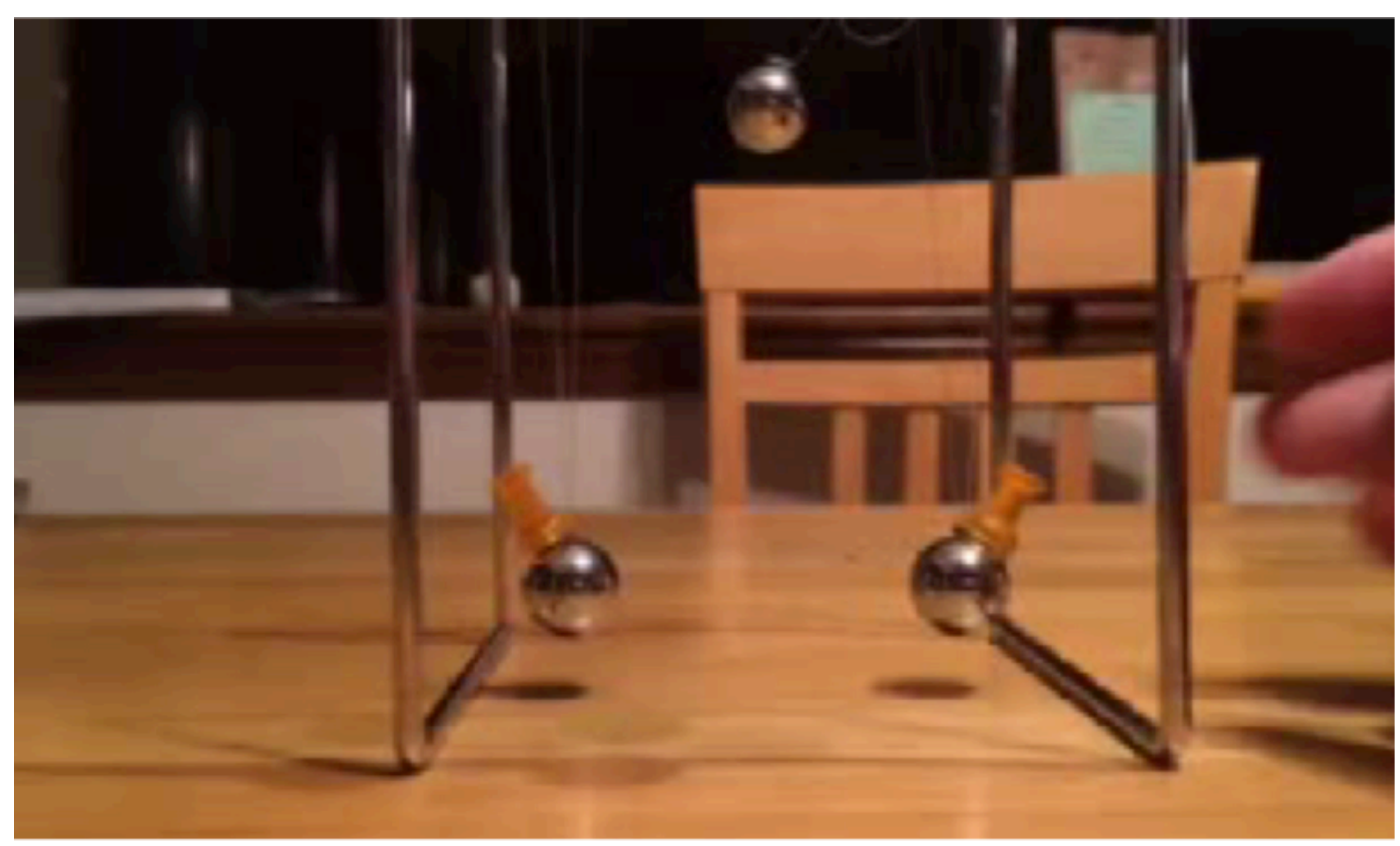

Weak coupling -- takes many swings to fully transfer the wave amplitude. Number of swings = cavity Quality factor.

Narrowband cavity response $\rightarrow$ iterative scan through frequency space. 


\section{Scaling laws}

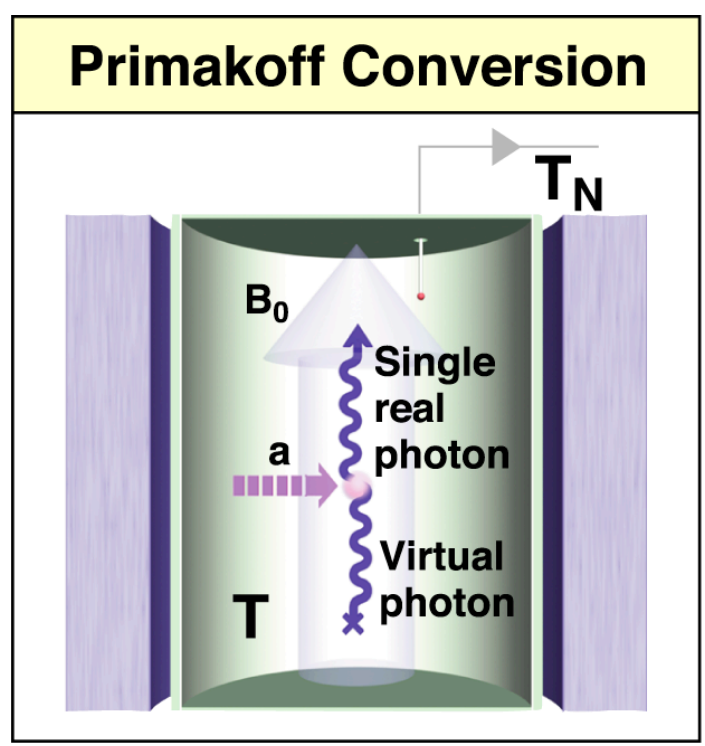

- The conversion is resonant, i.e. the frequency must equal the mass + K. E.

- The total system noise temperature $T_{S}=T+T_{N}$ is the critical factor

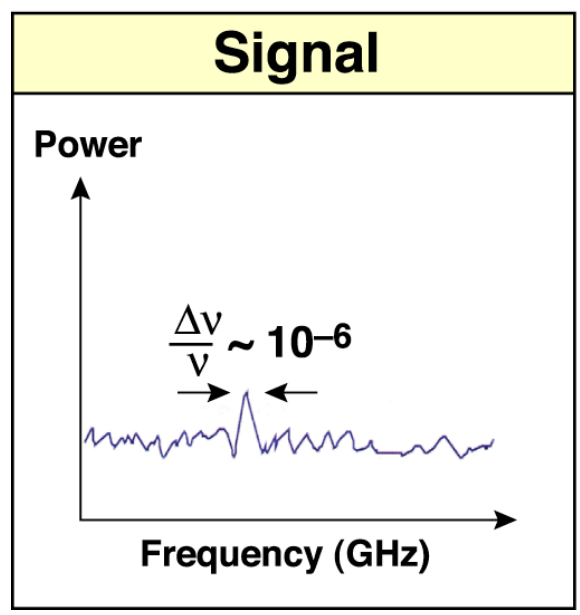

\begin{tabular}{|c|c|}
\hline \multicolumn{2}{|c|}{ Scaling Laws } \\
\hline$\frac{\mathbf{d} v}{\mathbf{d t}} \propto \mathbf{B}^{4} \mathbf{V}^{2} \cdot \frac{1}{\mathbf{T}_{S}^{2}}$ & $\mathbf{g}_{\gamma}^{2} \propto\left(\mathrm{B}^{2} \mathrm{~V} \cdot \frac{1}{\mathbf{T}_{\mathrm{S}}}\right)^{-1}$ \\
\hline For fixed model $\mathbf{g}^{2}$ & For fixed scan rate $\frac{\mathrm{d} v}{\mathrm{dt}}$ \\
\hline
\end{tabular}




\section{Axion current}

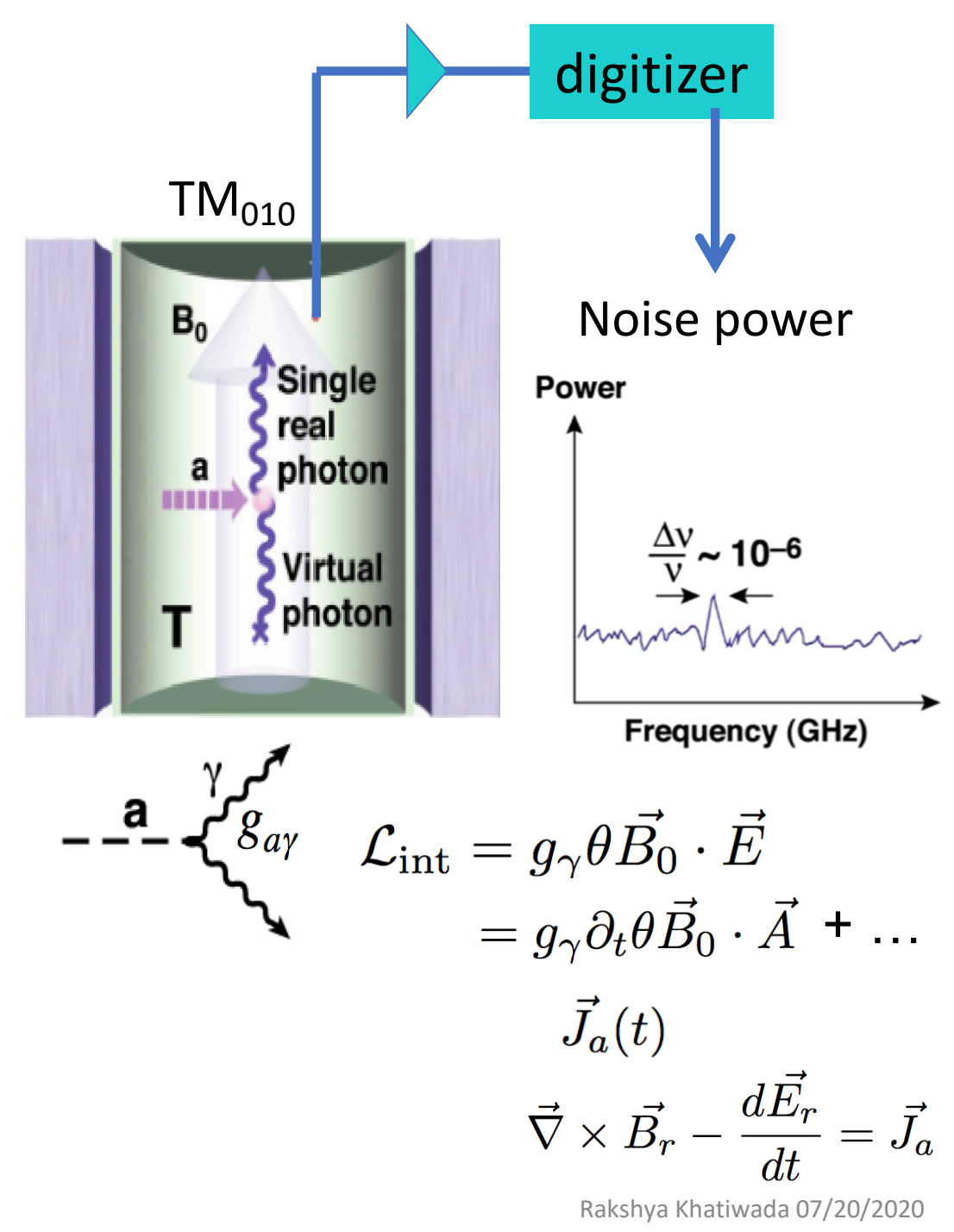

In a constant background $B_{0}$ field, the oscillating axion field acts as an exotic, space-filling current source

$\vec{J}_{a}(t)=-\frac{g \alpha}{\pi}\left(\frac{\sqrt{2 \rho_{a}}}{\Lambda_{\mathrm{QCD}}^{2}}\right) \vec{B}_{0} m_{a} e^{i m_{a} t}$

The Haloscope optimally extracts power from the potential energy of interaction:

$$
P_{a}(t)=\int \vec{J}_{a}(t) \cdot \vec{E}_{r}(t) d V
$$




\section{Cavity array}

- Higher frequency search:

$$
\begin{aligned}
& f=\frac{c}{2.61 * R} \text { or } \frac{R}{1 \mathrm{~cm}}=\frac{11.5 \mathrm{GHz}}{f} \\
& f=550 \mathrm{MHz} \Rightarrow R=21 \mathrm{~cm}, L=100 \mathrm{~cm} \\
& f=4.5 \mathrm{GHz} \Rightarrow R=2.6 \mathrm{~cm}, L=5.6 \mathrm{~cm}
\end{aligned}
$$

Cavities get smaller -- use many cavities $\square$ Need to be in phase/identical resonance

$$
\text { -- frequency lock system }
$$

$\square$ Power combiner and divider R \&D

\begin{tabular}{|l|l|l|l|}
\hline Cavities \# & $\begin{array}{l}\text { Res freq. } \\
\mathrm{MHz}\end{array}$ & $\begin{array}{l}\text { Tuning range } \\
\mathrm{MHz}\end{array}$ & $\begin{array}{l}\text { Tuning } \\
\text { range } \\
\mu \mathrm{eV}\end{array}$ \\
\hline 1 & 575 & $402-575$ & $1.7-2.4$ \\
\hline 1 & 575 & $575-908$ & $2.4-3.8$ \\
\hline 2 & 897 & $897-1417$ & $3.7-5.9$ \\
\hline 4 & 1207 & $1207-1907$ & $5-7.9$ \\
\hline 8 & 1899 & $1899-3001$ & $7.8-12$ \\
\hline 16 & 2959 & $2959-4675$ & $12-19$ \\
\hline 32 & 3983 & $3983-6293$ & $16-26$ \\
\hline
\end{tabular}

$\square>1 \mathrm{GHz}$ in production/development

\section{Cavities etc.:}

multi-array, photonic band-gap, open resonators, photon counting

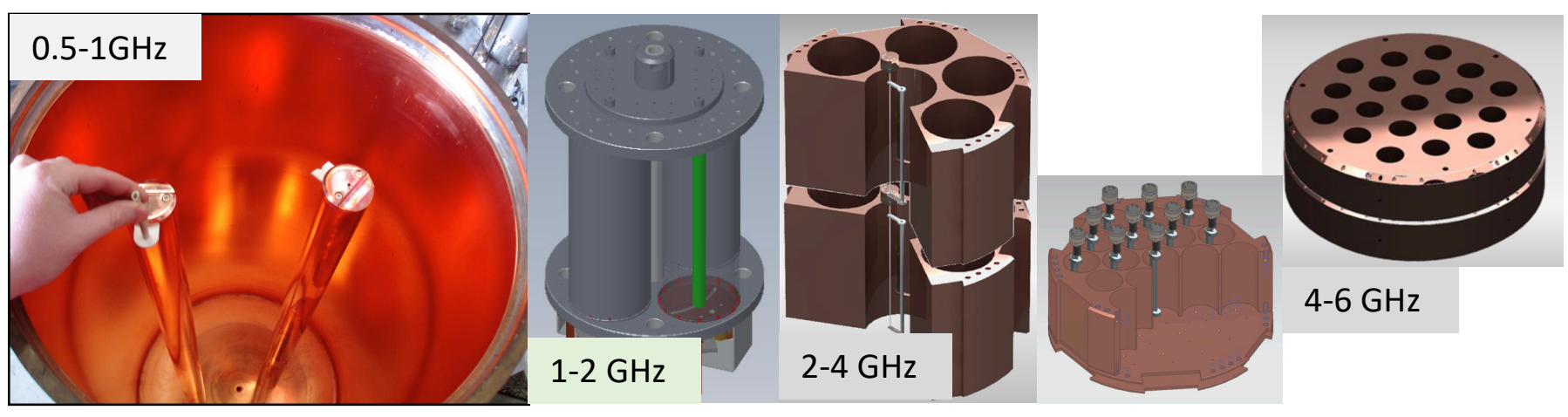




\section{Future technology for axion search}

- Multi-cavity array: power combine

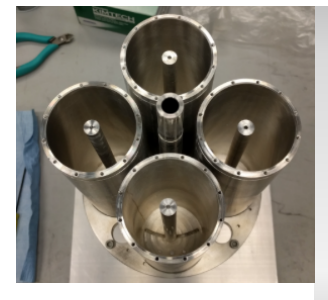

- Open resonators: resonators and series of current carrying wires (Orpheus etc.)

- Photonic bandgap cavities: Isolate a single mode using a defect in an open periodic lattice of metal and/or dielectric rods. High volume, defined mode

- Dielectric tuned cavity: lower loss/higher $\mathrm{Q}$ and form factor, $\mathrm{B}$ field compatible

- Quantum Non-Demolition (QND) photon

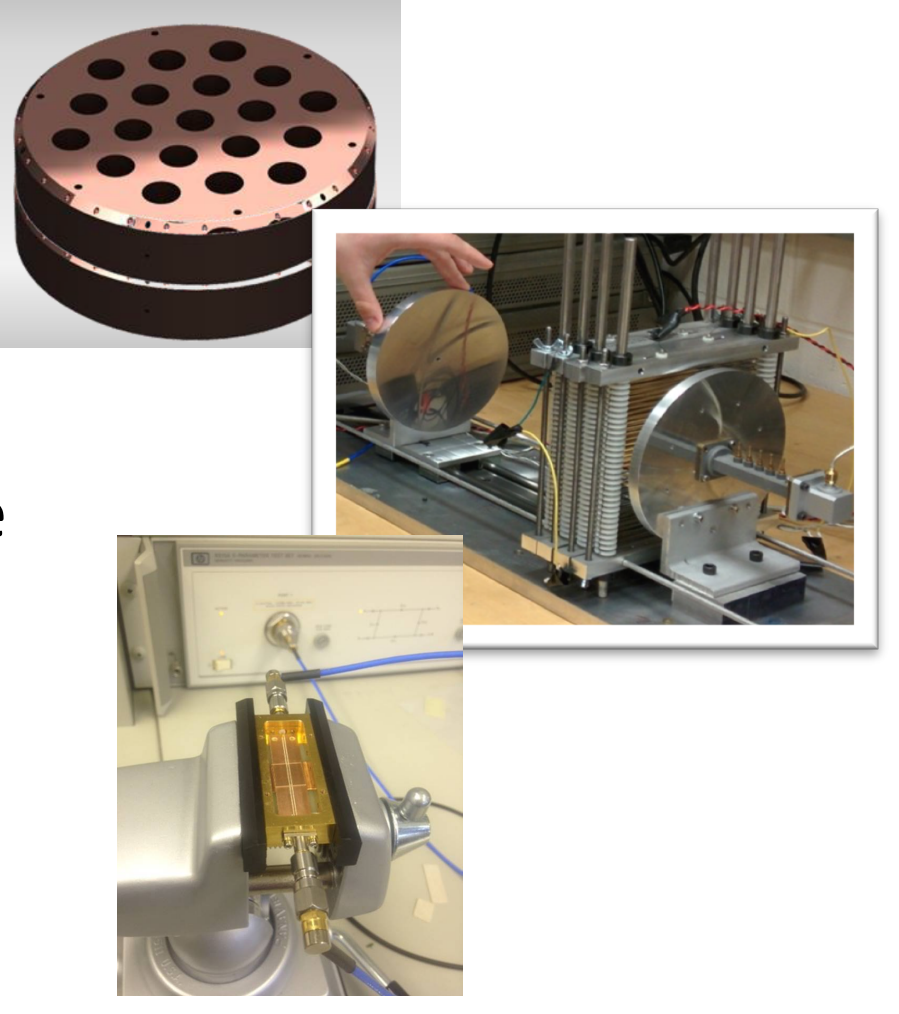
counting

- Squeezed parametric amplifier for $<$ QNL 


\section{Typical ADMX Run Cadence}

- Start by injecting a broad, swept RF signal to record cavity response. Record state data (temperatures, hall sensors, pressures, etc)

- Integrate for $\sim 100 \mathrm{sec}$ to $10 \mathrm{~s}$ of minutes (final integration time dependent experimental parameters).

- Every few days adjust the critical coupling of the antennas

- Scan rate is trade off in sensitivity vs frequency (mass) coverage

- The scan rate uses a threshold sensitivity.

- Any candidate above threshold is flagged for further study.

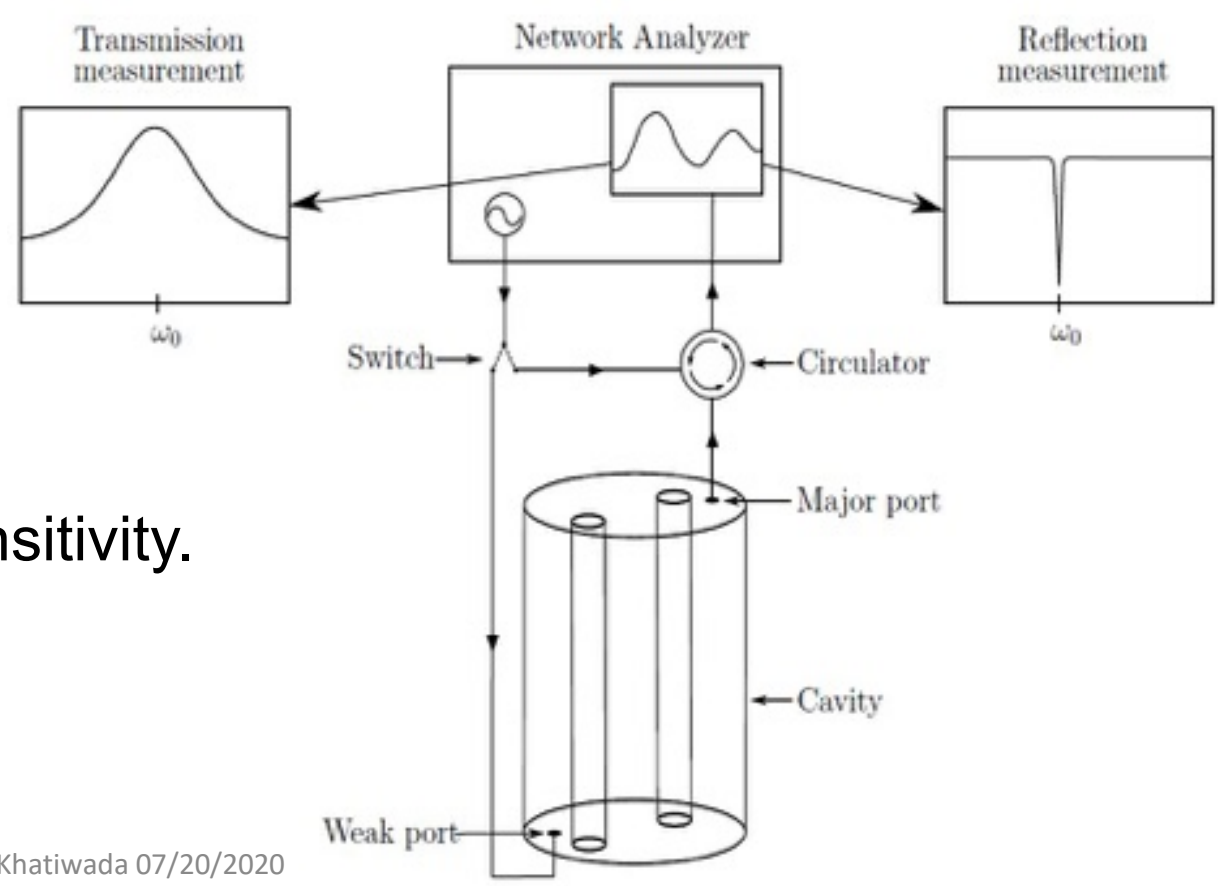




\section{Limitation of quantum amplifiers}

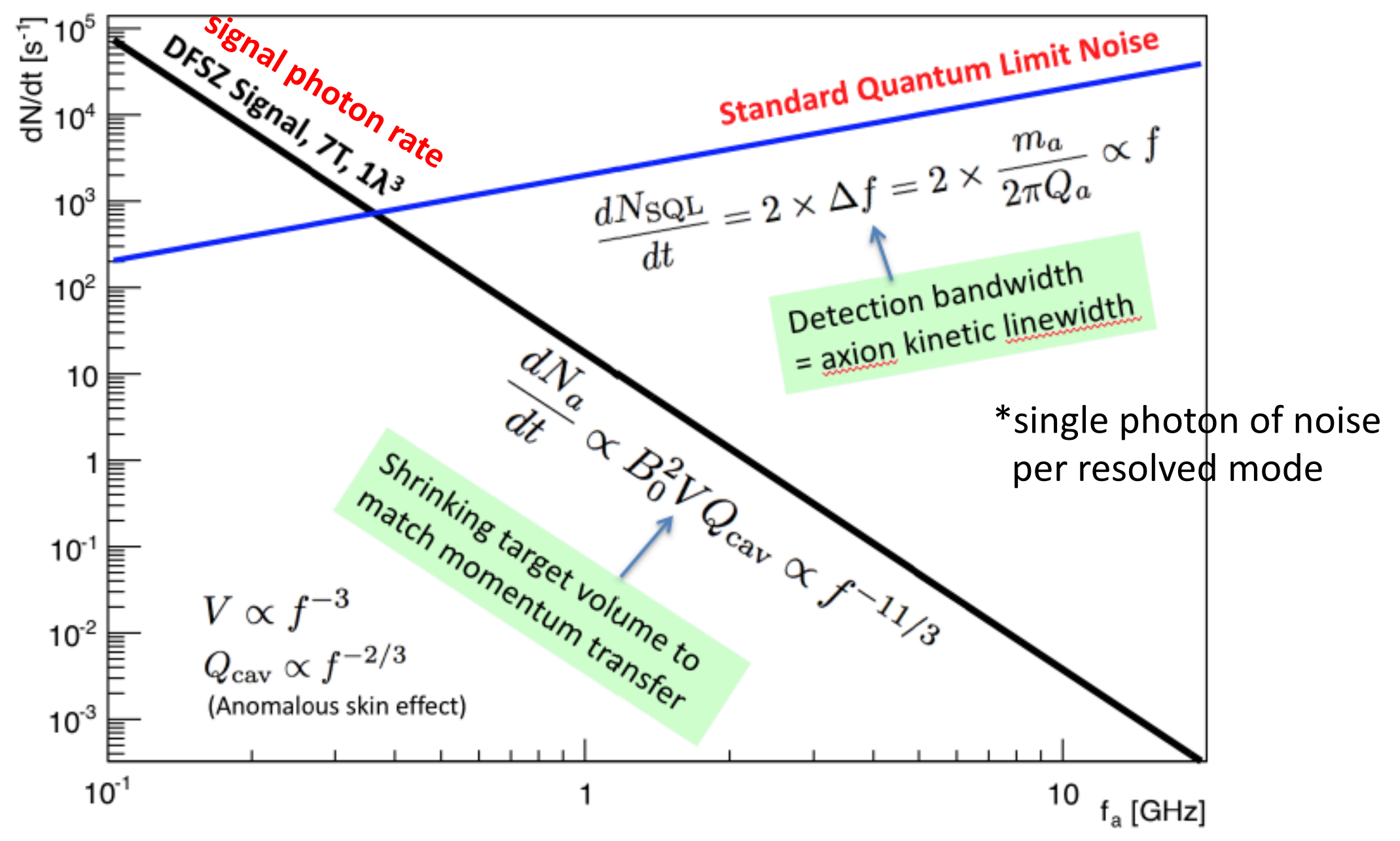




\section{Josephson Parametric amplifier (JPA)}

- Parametric amplifier: Oscillator whose resonance frequency is modulated

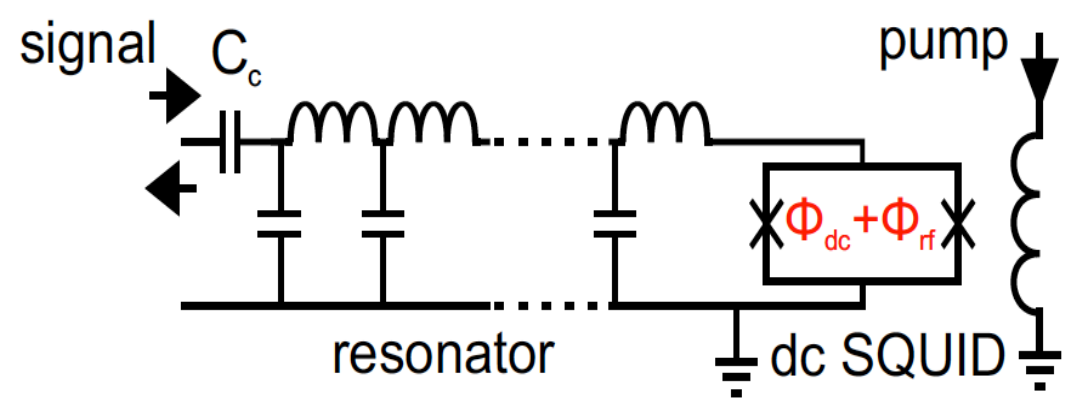

- Oscillating system a $\lambda / 4$ resonator

- Inductance varied with SQUID (flux dependent nonlinear inductor)

- Energy transfer from pump to two normal modes of swing

- Noise-Quantum Limit

$$
\omega_{0}=\frac{1}{\left.\left.2 \pi \sqrt{\left(C \left(L_{\text {stray }}\right.\right.}+L_{\text {SQUID }}\right)\right)}
$$

two normal modes of swing:

signal + idler

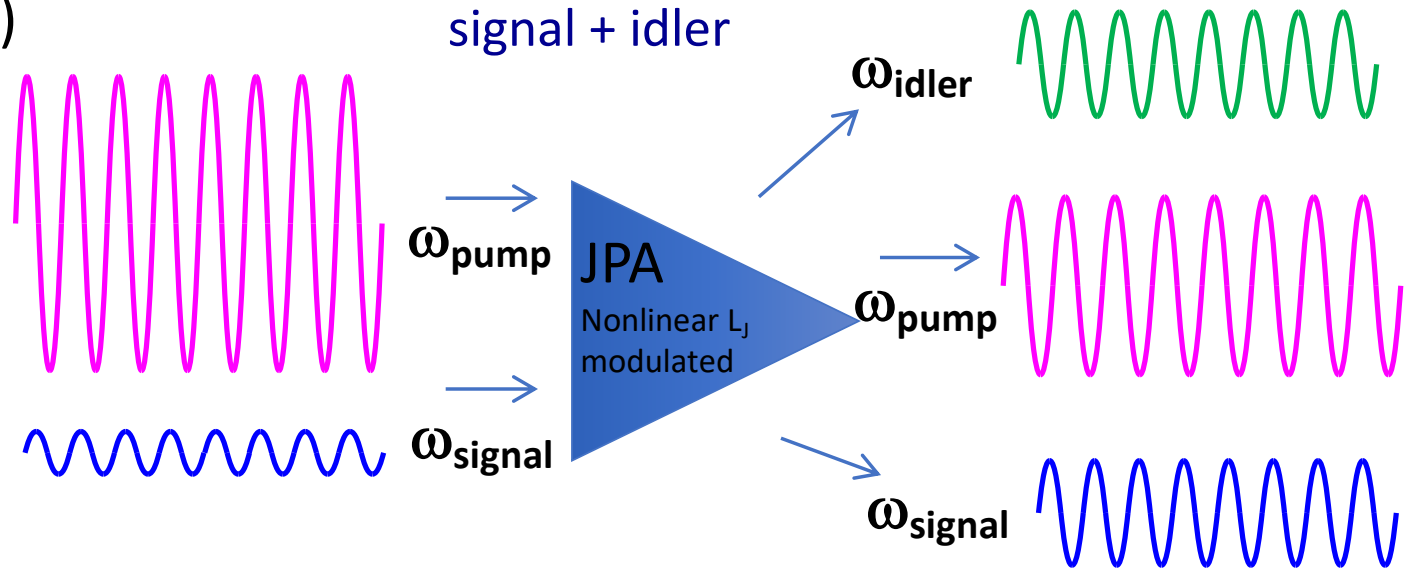




\section{Tuning the dark matter radio}

1. Tune the cavity and SQUID amps. to the desired frequency -- $\mathrm{m}_{\mathrm{a}}$

2. Achieve lowest system noise temp.

3. Record noise power spectra

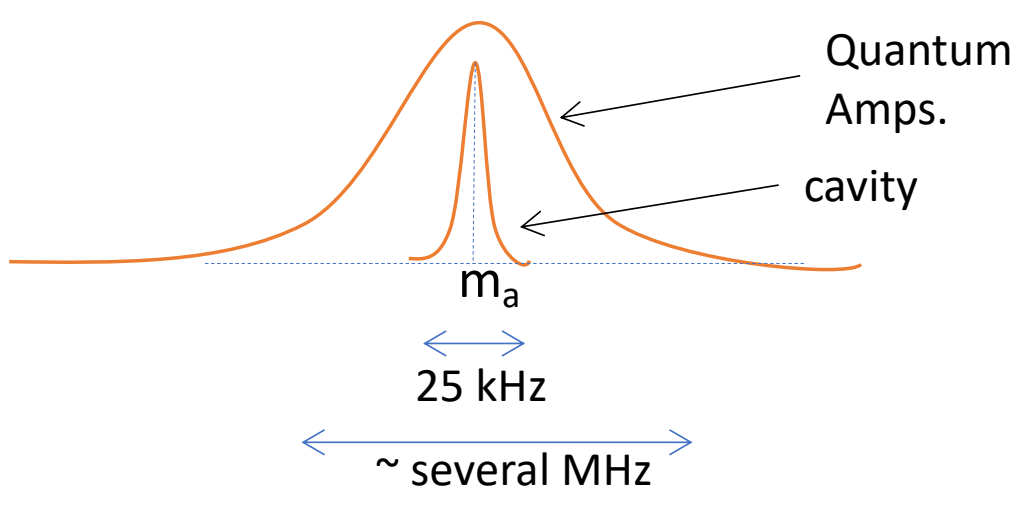

4. Digitize (100s)

5. Repeat until desired SNR

Repeat the above for different $m_{a}$

6. Analyze data -- filter, convolve with axion lineshape

7. Excess power signals rescanned

8. If candidate persist, Individually probe

9. Put limits or discover Axion! 